\title{
Análise temporal do aleitamento materno em Unidade Básica de Saúde de Pelotas, Brasil
}

\author{
Temporal analysis of breast-feeding in a Primary Health-care Unit of Pelotas, Brazil
}

\author{
Erick Dupont ${ }^{1}$, Martina Vitória Flach Dietrich ${ }^{1}$, Rafael Augusto Frizzo ${ }^{1}$, \\ Leonardo Elpídio Ribeiro ${ }^{1}$, Ângela Moreira Vitória ${ }^{2}$
}

Dupont E, Dietrich MVF, Frizzo RA, Ribeiro LE, Vitória AM. Análise temporal do aleitamento materno em Unidade Básica de Saúde de Pelotas, Brasil / Temporal analysis of breast-feeding in a Primary Health-care Unit of Pelotas, Brazil. Rev Med (São Paulo). 2017 jan.-mar.;96(1):35-8.

RESUMO: O conjunto de ações de atenção à saúde da criança implementado no eixo da assistência básica integral foi uma das determinantes da redução da mortalidade infantil no Brasil. Uma dessas ações foi o estímulo ao aleitamento materno que foi relacionado à prevenção de diversas doenças em lactantes e crianças. Por esse motivo, foi realizada uma pesquisa com método transversal descritivo com utilização de dados secundários em UBS no município de Pelotas - RS, com o objetivo de analisar temporalmente o aleitamento materno nos três primeiros meses de vida das crianças usuárias do serviço. Os dados foram obtidos das fichas espelho de puericultura da unidade e posteriormente agrupados em tabela confeccionada em Microsoft Excel 2013. A amostra foi composta por 246 crianças, nascidas entre 2011 e 2015. Não foi possível evidenciar um padrão temporal de melhora ou piora nos índices de aleitamento, uma vez que se verificou a inadequação de registros, contudo, inferiu-se que nos períodos de melhor registro, o percentual de aleitamento materno exclusivo também apresentou maiores valores. A média de aleitamento materno encontrada no período foi consonante com outros estudos realizados na Bahia, Florianópolis e Itapira (SP). No entanto, tais resultados ainda estão aquém do preconizado pela OMS, sendo verificada a necessidade de se instituir medidas que estimulem o aleitamento materno, além da realização de um melhor controle e avaliação dos serviços de saúde prestados pelo SUS na atenção primária.

Descritores: Aleitamento materno; Cuidado da criança; Atenção primária à saúde; Brasil.
ABSTRACT: Children's health care actions developed in basic health assistance was crucial to reduce infant mortality in Brazil. One of these actions, the stimulus breastfeeding, was associated with prevention of several diseases in children. Using a descriptive cross-sectional study from a secondary database, a research was done in order to analyze the breastfeeding in the first three months of life of infants users of the service provided by the Vila Municipal Basic Heath Unit at Pelotas, Rio Grande do Sul, Brazil. The data were gathered in Microsoft Excel 2013. The sample was 246 children born from 2011 to 2015. It was not possible to identify a temporal increasing or decreasing pattern of breastfeeding results, once the registration was inappropriately done. Nevertheless, during the period of more completed results, the breastfeeding also presented superior results. The medium of breastfeeding found was in alignment with other results find in Bahia, Florianópolis and Itapira (SP). Still they are down from what is implied by WHO. In fact, it is essential to lay down action to stimulate breastfeeding, also to establish better instruments of evaluation and control of the healthy services provided by SUS (Unit Healthy system) in primary attention.

Keywords: Breast feeding; Child care; Primary health care; Brazil.

Apresentação oral no Brazilian International Congress of Medical Students (BRAINCOMS), organizado pela UNIFESP, em 22 out. 2016.

1. Acadêmicos de Medicina da Universidade Federal de Pelotas. E-mail: dupont.erick@gmail.com, martinav.flach@gmail.com,r-frizzo@ hotmail.com, leonardoelpidio@live.com.

2. Orientadora. Professora assistente do Departamento de Medicina Social da Universidade Federal de Pelotas. E-mail: angela.vitoria@ ufpel.edu.br. 


\section{INTRODUÇÃO}

- egundo o $\mathrm{IBGE}^{1}$, no Brasil, na década de 80 , óbitos a cada mil nascidos vivos até um ano de idade. Visando a redução desses índices, foram criadas ações para a atenção à saúde da criança, as quais formaram o eixo da assistência básica integral. O conjunto dessas ações foi essencial para a melhora dos índices brasileiros de mortalidade infantil, sendo que, em 2009, segundo o IBGE ${ }^{1}$, essa taxa caiu para $18,79^{1}$. Dentre essas ações, prioriza-se a garantia da amamentação, a qual é fundamental para o desenvolvimento da criança.

Victora et al. ${ }^{2}$, através de revisões sistemáticas, mostra que a amamentação adequada previne a morbidade infantil por diarreia, infecções respiratórias e otite média, além de diminuir a mortalidade por causas como a enterocolite necrotizante e a síndrome da morte súbita na infância ${ }^{2}$. Além disso, evidencia-se a melhora do capital humano por meio do aumento da inteligência a longo prazo, a prevenção do câncer de mama nas lactantes, bem como prováveis efeitos de proteção sobre a ocorrência de excesso de peso e diabetes entre crianças amamentadas e no câncer de ovário e diabetes entre as mães. Não obstante, Figueiredo et al. ${ }^{3}$, em uma revisão de literatura, encontrou evidências de que o aleitamento materno (AE) protege as mães de depressão pós-parto, além de melhorar os padrões de sono e vigília da mãe e da criança. Nesse sentido, estima-se que a ampliação do aleitamento materno possa prevenir 823.000 mortes de crianças e 20.000 por câncer de mama a cada $a^{4}{ }^{4}$.

Com base nesse contexto, o Ministério da Saúde (MS) adotou o padrão preconizado pela Organização Mundial da Saúde (OMS), que estimula o aleitamento exclusivo até os 6 meses de idade, posteriormente, o aleitamento predominante/complementado até 2 anos de idade $^{5,6}$. Portanto, a unidade básica de saúde, representante da atenção primária do Sistema Único de Saúde brasileiro (SUS) é responsável por monitorar a amamentação para que esta seja realizada da maneira correta, além de instruir os pacientes sobre a importância do aleitamento exclusivo até os seis meses de idade para o bebê e também para a mãe.

Considerando a melhora dos indicadores de saúde da criança e a importância do aleitamento materno exclusivo para o desenvolvimento infantil, o presente trabalho objetiva avaliar os índices de amamentação dos usuários da UBS Vila Municipal, uma vez que a análise dos serviços de saúde é fundamental para garantir a qualidade da assistência prestada à população.

\section{METODOLOGIA}

O método utilizado foi o transversal descritivo com base nos dados secundários das crianças atendidas na UBS Vila Municipal, no período de janeiro de 2011 a dezembro de 2015. Para tanto, foi realizada a coleta de dados por quatro alunos do quarto semestre de medicina da Universidade Federal de Pelotas, a partir das fichas espelho de puericultura (FEP) da unidade. As FEP consistem em uma extensão do prontuário da criança, local onde devem ser registrados os mesmos dados da carteira de saúde da criança (CSC), com a única diferença de que esta permanece com a mãe e aquela é arquivada na unidade. Optou-se por este tipo de delineamento, visto que ele é o mais adequado para avaliar programas de saúde, além de ser o mais rápido e o de menor custo dentre os estudos observacionais.

A UBS Vila Municipal, está localizada no bairro Santos Dumont, no município de Pelotas, estado do Rio Grande do Sul, abrangendo uma população de, aproximadamente, 4.000 habitantes, divididos em 4 subáreas de atendimento do programa Estratégia da Saúde da Família. Seu funcionamento se dá a partir de uma parceria entre a Universidade Federal de Pelotas (UFPEL), Associação Beneficente Luterana de Pelotas (ABELUPE) e a Secretaria Municipal de Saúde de Pelotas.

Foram incluídas na amostra todas as fichas espelho cadastrada na UBS no período supracitado, totalizando uma amostra de 246 indivíduos. Com base nas informações dessas fichas espelho e também em uma planilha de coleta de dados de puerpério criada pelo Departamento de Medicina Social da Universidade Federal de Pelotas, foi confeccionada uma tabela própria em Microsoft Office Excel 2013 para a tabulação dos dados. As variáveis analisadas foram: aleitamento exclusivo, aleitamento predominante e uso de outro leite, as quais foram comparadas ao longo dos anos, considerando apenas os três primeiros meses de vida da criança. A análise do aleitamento até os 6 meses como preconizado pela OMS não foi possível devido à quantidade insuficiente de dados do $4^{\circ}, 5^{\circ}$ e $6^{\circ}$ mês.

\section{RESULTADOS}

A amostra foi composta por 246 crianças, sendo 22 nascidas em 2011, 46 em 2012, 48 em 2013, 95 em 2014 e 35 em 2015. Como demonstrado no Gráfico 1, o ano de 2014 obteve o maior percentual de registro no primeiro mês $(84,2 \%)$, sendo o aleitamento materno exclusivo o tipo de nutrição prevalente (60\%). Enquanto isso, no segundo mês, os dois anos com melhores registros mostraram as maiores porcentagens de aleitamento materno exclusivo e uso de outro leite, enquanto os dois anos com menos registro mostraram as menores porcentagens dessas variáveis. No terceiro mês, este padrão foi mantido, excetuando-se 2011, que teve o menor número de registros (50\%), mas apresentou o maior percentual de AE (27,3\%). 
Gráfico 1: Padrão de amamentação ao longo dos anos estratificado por idade (Análise temporal do aleitamento materno em unidade básica de saúde de Pelotas, Brasil)

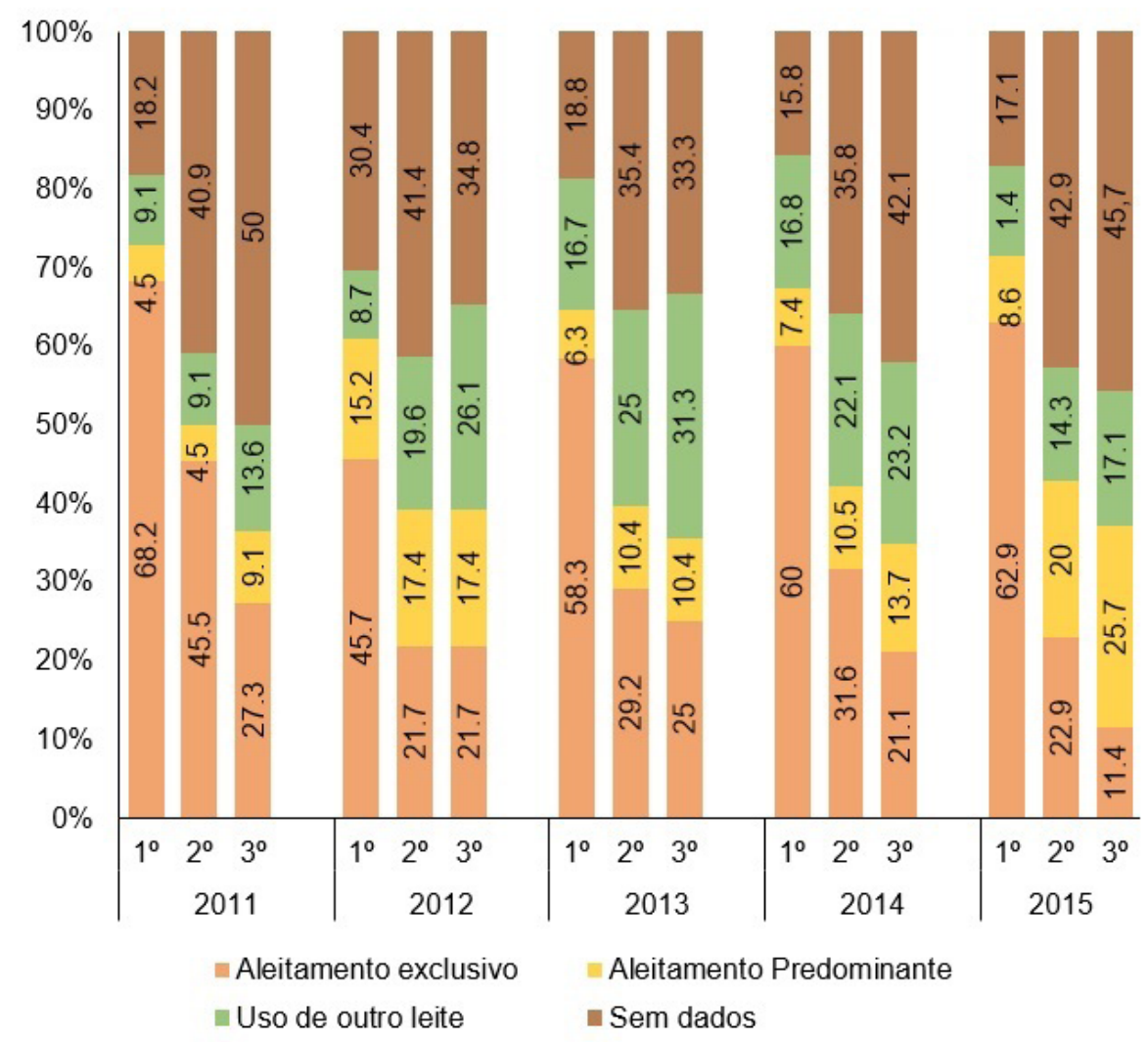

Fonte: autores, 2016.

Nesse contexto, a análise temporal não evidenciou um padrão de melhora ou piora dos índices de amamentação, já que o registro inadequado inviabilizou tal conclusão. Entretanto, é possível inferir que os anos nos quais o registro é melhor, o percentual de aleitamento materno exclusivo e uso de outro leite são maiores.

O Gráfico 2 estratifica as prevalências de cada tipo de alimentação nos meses analisados. No primeiro mês, a média de prevalência de aleitamento materno exclusivo de 2011 a 2015 foi de 58,8\%, sendo que 2011 e 2015 mostraram os melhores resultados, alcançando $68,2 \%$ e $62,9 \%$, respectivamente. Em contrapartida, o ano de 2012 obteve a menor porcentagem de dessa variável $(45,7 \%)$ no primeiro mês.

\section{DISCUSSÃO}

Esses resultados são similares aos encontrados por Vieira et al. ${ }^{7}$ na Bahia, onde a média de prevalência de aleitamento materno exclusivo no primeiro mês foi de $59,3 \%{ }^{7}$. O mesmo é visto no segundo e terceiro mês, em que os índices de desse tipo de aleitamento diminuem enquanto os de aleitamento predominante e uso de outro
Gráfico 2: Média do tipo de aleitamento materno nos três primeiros meses de vida. (Análise temporal do aleitamento materno em unidade básica de saúde de Pelotas, Brasil)

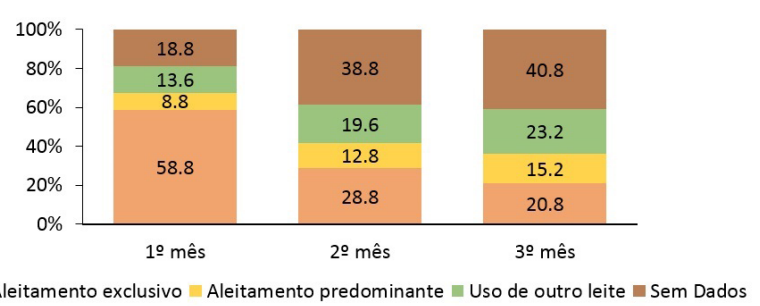

Fonte: autores, 2016.

leite aumentam, alcançando prevalências mais altas do que aleitamento materno exclusivo no terceiro mês de 2015. No mesmo sentido, estudo realizado em Florianópolis mostrou que $60 \%$ das crianças receberam leite exclusivamente da mãe no primeiro mês e que esse índice sofreu uma redução para menos de $20 \%$ quando analisado até o sexto mês de vida $^{8}$.

Em Itapira - SP, inquérito populacional também encontrou índices semelhantes: a prevalência do aleitamento materno exclusivo no primeiro mês foi de $64,8 \%$, caindo 
para $45 \%$ aos quatro meses e $30,1 \%$ aos seis ${ }^{9}$. Esse estudo também verificou que a interrupção do aleitamento materno exclusivo nos primeiros seis meses estaria relacionada com o uso de chupeta e a realização de parto cesáreo.

Revisão sistemática realizada por Oliveira e Camacho ${ }^{10}$ concluiu que a promoção, proteção e o apoio à amamentação, realizada a nível de atenção primária, contribui para intensificar a prática do aleitamento materno exclusivo até os 6 meses. As intervenções que se mostraram efetivas nesse estudo foram: ouvir as queixas das mães e fornecer orientações para superar as dificuldades; informar sobre a importância de se iniciar a amamentação precocemente; informar sobre suas vantagens; orientar sobre os riscos do uso de outro leite, mamadeiras e chupetas; orientação quanto à pega, posição e ordenha; e orientação para contracepção.

\section{REFERÊNCIAS}

1. IBGE. Censo demográfico 2010 - Resultados gerais da amostra. Rio de Janeiro; 2012. Disponível em: http://www. ibge.gov.br/home/presidencia/noicias/imprensa/ppts/000000 08473104122012315727483985.pdf.

2. Victora CG, Bahl R, Barros AJ, França GV, HORTON S, Krasevec J, Murch S, Sankar MJ, Walker N, Rollins NC. Breastfeeding in the 21st century: epidemiology, mechanisms, and lifelong effect. Lancet. 2016;387(10017):475-90. doi: http://dx.doi.org/10.1016/S0140-6736(15)01024-7.

3. Figueiredo B, Dias CC, Brandão S, Canário C, Nunes-Costa R. Breastfeeding and postpartum depression: state of the art review. J Pediatr (Rio J). 2013;89(4):332-8. doi: http://dx.doi. org/10.1016/j.jped.2012.12.002.

4. Requejo JH, Bryce J,Barros AJ, Berman P, Bhutta Z, Chopra M, Daelmans B, de Francisco A, Lawn J, Maligi B, Mason E, Newby H, Presern C, Starrs A, Victora CG. Countdown to 2015 and beyond: fulfilling the health agenda for women and children. Lancet. 2015;385:466-76. doi: http://dx.doi. org/10.1016/S0140-6736(14)60925-9

5. Brasil. Ministério da Saúde. Saúde da criança: crescimento e desenvolvimento. Brasília; 2012. (Cadernos de atenção básica, n.33). Disponível em: http://dab.saude.gov.br/portaldab/ biblioteca.php?conteudo=publicacoes/cab33.

6. World Health Organization (WHO). Infant and young child

\section{CONCLUSÕES}

A puericultura, instaurada no âmbito da atenção primária, foi essencial para a melhoria da saúde da população infantil nas últimas décadas. Nesse contexto, surge a importante contribuição do aleitamento materno para essa conquista. De acordo com os resultados obtidos neste estudo, evidencia-se que ainda não foram atingidos os parâmetros preconizados pela $\mathrm{OMS}^{6}$ em relação à amamentação. Por essa razão, faz-se necessária maior atenção a essa prática, com a correta instrução das mães e dos profissionais de saúde acerca de sua importância. Além disso, verifica-se a necessidade de controle e avaliação dos programas de saúde, para que os problemas possam ser identificados e resolvidos, a partir da elaboração de estratégias que visem à melhoria das condições de saúde da população.

feeding - model chapter for textbooks for medical students and allied health professionals. Geneva; 2009. Available from: http:// apps.who.int/iris/bitstream/10665/44117/1/9789241597494_ eng.pdf?ua $=1 \& u a=1$.

7. Vieira GO, Martins CC, Vieira TO, Oliveira NF, Silva LR. Fatores preditivos da interrupção do aleitamento materno exclusivo no primeiro mês de lactação. J Pediatr (Rio J.). 2010;86(5):441-4. doi: http://dx.doi.org/10.1590/S002175572010000500015 .

8. Carvalho M, Lira P, Romani S, Santos IS, Veras AACA, Batista Filho M. Acompanhamento do crescimento em crianças menores de um ano: situação nos serviços de saúde em Pernambuco, Brasil. Cad Saúde Pública (Rio J). 2008;24(3):675-85. doi: http://dx.doi.org/10.1590/S0102311X2008000300021.

9. Audi CAF, Correa MAS, Latorre MRDO. Alimentos complementares e fatores associados ao aleitamento materno e ao aleitamento materno exclusivo em lactentes até 12 meses de vida em Itapira, São Paulo, 1999. Rev Bras Saude Matern Infant (Recife). 2003;3(1):85-93. doi: http://dx.doi. org/10.1590/S1519-38292003000100011.

10. Oliveira MIC, Camacho LAB. Impacto das Unidades Básicas de Saúde na duração do aleitamento materno exclusivo. Rev Bras Epidemiol. 2002;5(1):41-51. doi: http://dx.doi. org/10.1590/S1415-790X2002000100006. 